\title{
Relación entre autoestima y autoconfianza matemática en estudiantes de educación media costarricense
}

\author{
Por: Dra. Evelyn Agüero-Calvo ${ }^{1}$, Dra. Martha Calderón-Ferrey ${ }^{2}$, Dr. Luis \\ Gerardo Meza-Cascante ${ }^{3}$ y Dra. Zuleyka Suárez-Valdés-Ayala ${ }^{4}$, Instituto \\ Tecnológico de Costa Rica
}

$\begin{array}{ll}\text { Fecha de recibido: } & 7 \text { de junio, } 2016 . \\ \text { Fecha de aceptación: } & 6 \text { de setiembre, } 2016 .\end{array}$

\section{Resumen}

En este artículo se exponen los resultados de un estudio acerca de la relación entre la autoestima y la autoconfianza matemática de quienes están cursando la educación media costarricense. En dicho estudio se indagó sobre la existencia de diferencias entre hombres y mujeres en ambos constructos. El estudio se realizó con una muestra de 2984 estudiantes de colegios públicos diurnos oficiales del Ministerio de Educación Pública de Costa Rica, durante el año 2015. Los resultados sugieren que, en forma conjunta, $78.9 \%$ del estudiantado muestra niveles de autoconfianza entre alto y moderado y $74.4 \%$ presenta niveles de autoestima entre alto y moderado. También se encontró que existen diferencias estadísticamente significativas tanto en el nivel de autoconfianza como en el nivel de autoestima según el sexo, con niveles menores para las mujeres. Finalmente, la investigación reveló la existencia de una relación lineal significativa entre el nivel de autoconfianza matemática y el de autoestima.

\section{Abstract \\ Relationship between self-esteem and mathematical self-confidence in high school students in Costa Rica \\ This paper presents the results of a study about the relationship between self-esteem and mathematical self-confidence of students in Costa Rican high schools. Such study shows research upon the existence of differences between men and women in regards to both constructs. The study was carried out with a sample of 2984 students of public, daytime official high schools of the Ministry of Public Education of Costa Rica during 2015. The results suggest that, as a whole, $78.9 \%$ of the students show levels of self- confidence between high and moderate; $74.4 \%$ show levels of self-esteem between high and moderate. It also was found that there are statistically significant differences between the level of self-confidence, as well as in the self-esteem according to sex, with lower levels in the case of women. Finally, the research showed the existence of a linear relation between the level of mathematical self-confidence and that of self- esteem.}

1 Evelyn Agüero es doctora en Intervención Educativa por la Universidad de Valencia, España, Máster en Matemática Educativa por el CINVESTAV de México y Bachiller en la Enseñanza de la Matemática asistida por Computadora por el Instituto Tecnológico de Costa Rica. Profesora Intermedio Catedrática e Investigadora 1 en el Instituto Tecnológico de Costa Rica. Contacto: evaguero@itcr.ac.cr.

2 Doctora en Intervención Educativa por la Universidad de Valencia, España, Doctora en Derecho por la Universidad Estatal a Distancia (UNED), Licenciada en Derecho por la Universidad de Costa Rica. Profesora Catedrática del Instituto Tecnológico de Costa Rica. Contacto: micalderon@itcr.ac.cr.

3 Doctor en Educación por la Universidad Estatal a Distancia, Máster en Administración de Empresas por el Instituto Tecnológico de Costa Rica y Licenciado en la Enseñanza de la Matemática por la Universidad Nacional. Profesor Catedrático e Investigador Consolidado 1 en el Instituto Tecnológico de Costa Rica (TEC). Contacto: gemeza@itcr.ac.cr.

4 Doctora en Educación por la Universidad Estatal a Distancia, Master en Educación y Licenciada en la Enseñanza de la Matemática por la Universidad Nacional. Profesora Asociada e Investigadora 1 en la Escuela de Matemática del Instituto Tecnológico de Costa Rica. Contacto: zsuarez@itcr.ac.cr.
Evelyn Agüero-Calvo, Martha CalderónFerrey, Luis Gerardo Meza-Cascante y Zuleyka Suárez-Valdés-Ayala. Relación entre autoestima y autoconfianza matemática en estudiantes de educación media costarricense. Revista Comunicación. Año 37, volumen 25, número 2, julio - diciembre, 2016. Instituto Tecnológico de Costa Rica. ISSN: 0379-3974 / e-ISSN1659-3820.

PALABRAS CLAVE:

Matemática, autoconfianza matemática, confianza en sí mismo, autoestima, angustia, enseñanza secundaria.

KEY WORDS:

Mathematics, mathematical self-confidence, self-security, self-esteem, anguish, high school education. 


\section{INTRODUCCIÓN}

En este artículo se reportan los resultados de una investigación acerca de la relación entre la autoestima y la autoconfianza matemática de quienes fueron parte de la educación media costarricense durante el año 2015 en colegios públicos diurnos oficiales. Las respuestas afectivas que evidencian los estudiantes al tener que lidiar constantemente con la presión ejercida sobre ellos con respecto a su rendimiento académico en matemática, juegan un papel fundamental en el proceso de enseñanza y aprendizaje de esta asignatura.

Durante varios años, los estudios sobre la dimensión afectiva en el aprendizaje de la matemática se limitaron al estudio de las actitudes. Sin embargo, en las últimas décadas, el estudio se amplió para incluir las creencias y las reacciones emocionales.

Este nuevo enfoque de la dimensión afectiva en el aprendizaje de la matemática pone de manifiesto que las cuestiones afectivas desempeñan un papel esencial en el proceso de enseñanza-aprendizaje de la matemática, y que algunas de ellas aparecen fuertemente arraigadas en las personas y no son fácilmente desplazables por el proceso de enseñanza (Gil, Blanco y Guerrero, 2006). De esta forma, resulta importante identificar aquellos aspectos que tienen influencia tanto positiva como negativamente en ese proceso.

La comunidad investigadora es consciente de la influencia de los factores afectivos en el aprendizaje de la matemática. Por este motivo, en los últimos años se ha incrementado el número de trabajos que profundizan esta temática (Gómez-Chacón, 2010). El estudio de estos factores en la educación secundaria es especialmente importante porque de acuerdo con Aschcraft (2005, citado por Primi, Busdraghi, Tomasetto, Morsanyi y Chiesi, 2014, p. 51), "el interés y la motivación declinan conforme el estudiante va creciendo, y la ansiedad matemática se piensa que se desarrolla en la educación secundaria, coincidiendo con la creciente dificultad del plan de estudios de matemáticas". Las creencias de autoeficacia tienen efectos positivos en los procesos motivacionales y volitivos, y en el rendimiento resultante; por otra parte, la ansiedad hacia los exámenes ha evidenciado efectos perjudiciales sobre el rendimiento (Schnell, Ringeisen, Raufelder y Rohrmann, 2015).
Aunque se han dado varias definiciones del constructo "autoconfianza matemática", con distintos niveles de equivalencia entre sí, se asume la planteada por Pérez-Tyteca (2012), que considera las aportadas por Fennema y Sherman (1976, citado por Pérez-Tyteca, 2012) y McLeod (1992, citado por Pérez-Tyteca, 2012).

La "autoconfianza matemática" se define como una creencia sobre la propia competencia matemática que consiste en la confianza que la persona tiene en sus propias habilidades para enfrentarse a tareas relacionadas con las matemáticas (Pérez-Tyteca, 2012). De esta manera, la "autoconfianza matemática" es una variable afectiva de gran importancia en las investigaciones en matemática educativa, centrándose los estudios de las últimas décadas en la búsqueda de sus causas, en los patrones de su evolución y en la relación con factores como el rendimiento académico, el género y la elección de cursos (Reyes, 1984, citado por Pérez-Tyteca, 2012). Además, Pérez-Tyteca, Monje y Castro (2013) encontraron en su estudio que en el paso del colegio a la universidad la variable "autoconfianza matemática" tiene efecto sobre la elección de la carrera universitaria.

Uno de los principales factores estudiados en relación con la autoconfianza en matemática, es diferencia de género (Pérez-Tyteca, 2012). Brown y Josephs (2001, citados por Gamboa, 2012), señalan que hombres y mujeres difieren en sus niveles de autoconfianza matemática. Pérez-Tyteca (2012) reseña con base en Sherman (1983), Reyes (1984) y McLeod (1992) que las investigaciones realizadas señalan la existencia de diferencias de género en el nivel de autoconfianza matemática, al punto que las mujeres aparecen con niveles menores, aun cuando no tengan razones para ser menos autoconfiadas ya que su desempeño académico no es inferior. Gamboa (2012) considera que esta diferencia se deba posiblemente a estereotipos sociales sobre las diferencias de género en matemática (los hombres son "buenos" en esta disciplina y las mujeres son "deficientes") o al hecho de que los estereotipos sociales y otros factores inhiben a las mujeres a tomar tantos cursos de matemáticas como lo hacen los hombres.

Contraponiendo los conceptos de autoestima y autoconcepto, Rabell (2012) afirma que este último es el conocimiento que el individuo tiene de sí mismo 
y por tanto en este se sustenta la autoestima, la cual es "un sentimiento de aceptación hacia uno mismo unido a un sentimiento de valía personal. Este sentimiento varía durante el curso de la vida, dependiendo de las distintas experiencias" (p. 9).

Puede afirmarse que el autoconcepto es el todo, pues está referido al conocimiento que el individuo tiene de sí mismo, mientras que la autoestima es una parte de ese todo, por cuanto está referida a la estimación que, a partir de sus vivencias y valores, hace el individuo del conocimiento que de sí mismo tiene (Calderón, 2015). García (2002) ha definido la autoestima "como el amor hacia sí mismo, aprecio, aceptación, valoración que se tiene de sí mismo, y que se manifiesta a través de su comportamiento en los diversos escenarios de la vida diaria" (p. 19). De esta manera, el medio en el cual se desarrolla el individuo influye en la autoestima, pues es de este de donde adopta su escala de valores y es reflejo de la adaptación del individuo a ese medio (Calderón, 2015).

\section{La autoestima es un proceso mediante el cual la persona, confronta sus acciones, sus sentimientos, sus capacidades, limitaciones y atributos en comparación a los criterios y valores personales que ha interiorizado, para valorarse a sí mismo. Es la confianza de la persona en su ser, basado en el conoci- miento real de sus posibilidades y potencia- lidades, fortalezas y debilidades, en el poder de sus convicciones y su energía, vigor y su fortaleza espiritual. La autoestima es la ex- periencia de ser competentes para enfrentar- se a los desafíos básicos de la vida, y de ser dignos de felicidad (Erazo, 2013, p. 21).}

De esta manera, la autoestima es el resultado de un proceso y no una variable genética. Las experiencias de vida del individuo, la capacidad para enfrentar retos y sobreponerse a las adversidades y la opinión que de él o de ella tengan las personas cercanas como padres, maestros, compañeros y amigos así son los elementos que entran en juego en la formación de la opinión que tenga de sí la persona y cómo construya su propia conducta y personalidad (Calderón, 2015; Erazo, 2013, p. 21).

La autoestima está compuesta por un conjunto de aspectos interrelacionados entre sí, lo que ha per- mitido que distintos autores procuren identificar los componentes de la autoestima. Como lo ha hecho Olivares (1997, citado por Naranjo, 2007), quien reseña lo siguiente:

a) La actitud: contempla las formas habituales de pensar, actuar, amar y sentir de las personas para consigo mismas.

b) El conocimiento: se refiere a las ideas, opiniones, creencias, percepciones y procesamiento de la información que posee la persona respecto de sí misma.

c) La afectividad: es la valoración de lo positivo y negativo, involucra sentimientos favorables y desfavorables, agradables o desagradables que las personas perciben de sí mismas.

d) La conducta: es la intención y decisión de actuar, de llevar a la práctica un comportamiento consecuente y coherente (Naranjo, 2007, p. 3).

Córdova (2010), por su parte, reduce los componentes del concepto de autoestima a tres, según el siguiente detalle:

a) Cognitivo: que indica idea, opinión, creencias, percepción procesamiento de la información.

b) Afectivo: conlleva la valoración de lo que en nosotros hay de positivo y de negativo, implica un sentimiento de lo favorable o desfavorable, que siente la persona de sí misma.

c) Conductual: se refiere a la tensión, intención y actuación que hace la persona por sí misma (p. 21).

A partir de los componentes de la autoestima señalados por Córdova (2010), se puede sostener que la autoestima contribuye a desarrollar la capacidad de superar los obstáculos, promueve la creatividad, fortalece la autonomía personal y contribuye al establecimiento de relaciones sociales saludables (Calderón, 2015). La autoestima es la valoración que de sí mismo hace el individuo y se pueden identificar dos dimensiones: la dimensión actitudinal y la dimensión conductual.

En la dimensión actitudinal se encierra la autoimagen, la autovaloración y la autoconfianza; en tanto que la dimensión conductual abarca el autocontrol, la autoafirmación y la autorealización. 
Para comprender la dimensión actitudinal que, como se ha adelantado, es inferida a partir de la conducta del individuo, se deben desarrollar sus componentes, a saber:

a) La autoimagen: es la capacidad del individuo de verse a sí mismo como realmente es, con sus virtudes y defectos. El individuo con alta autoestima es consciente de sus errores, defectos, virtudes y aciertos, pues su autoestima no se vincula con la perfección. "La autoimagen no es un aspecto estático, incluye el conocimiento propio basado en experiencias pasadas, al igual que las concepciones que la persona va elaborando sobre su posible evolución futura" (Mezerville, 2004, citado por Naranjo, 2007, p. 31).

b) La autovaloración: es la importancia que la persona se da a sí misma, tanto en relación con ella como en relación con los otros individuos que componen su entorno cotidiano. "La autovaloración se relaciona con otros aspectos como la autoaceptación y el auto respeto y significa que se percibe con agrado la imagen que la persona tiene de sí" (Naranjo, 2007, p. 10).

c) La autoconfianza: es el reconocimiento del individuo en relación con su derecho a tener éxito, a ser respetado y a satisfacer sus necesidades, así como la certeza de ser digno y de alcanzar sus principios éticos y obtener fruto de sus esfuerzos.

Una amplia mayoría de investigadores (Mezerville, 2005; Naranjo, 2007; Córdova, 2010, Rabell, 2012) se inclinan a considerar que la autoestima es un condicionante del éxito académico, afirmando que las y los estudiantes con una autoestima más alta se encuentran en mejor disposición de aprender, pues confían en sus cualidades y en su capacidad para vencer obstáculos y superar fracasos. Esta certeza ha llevado a muchos especialistas a estudiar y documentar sus hallazgos en cuanto a la relación entre la autoestima y el rendimiento académico (Calderón, 2015). Beane (1986) indica que "los estudios han demostrado una relación persistente entre el autoconcepto y la autoestima y diversas variables relacionadas con las actividades académicas" (citado por Naranjo, 2007, p. 7).
Si se acepta que la conducta revela la autoestima, debe sostenerse como lo afirman Fernández, Martínez-Conde y Melipillán (2009), que los y las estudiantes que utilizan estrategias de aprendizaje complejas, se involucran más en tareas de aprendizajes y manifiestan un mayor nivel de perseverancia y de esfuerzo, porque se trata de individuos con capacidad de autorealización y en consecuencia, presentan también, altos sentimientos de autoestima académica, frente a quienes utilizan estrategias de aprendizaje simples (Calderón, 2015).

En el campo de la educación matemática, los trabajos de McLeod (1992) y Mandler (1989), ampliamente referenciados, pusieron de manifiesto que las cuestiones afectivas y emocionales juegan un papel esencial en la enseñanza y aprendizaje de la matemática y, en particular, en la resolución de problemas. No obstante, De Bellis y Goldin (2006) y Furinghetti y Morselli (2009) recuerdan que tradicionalmente las investigaciones se han centrado, primeramente, en aspectos cognitivos, segundo en aspectos afectivos, pero pocas veces en la interacción de los aspectos cognitivos y afectivos. Sin embargo, cada vez son más los trabajos que reconocen la importancia de considerar las dimensiones afectiva y cognitiva de manera integrada en la enseñanza y aprendizaje de las Matemáticas (Amato, 2004; Blanco, Guerrero, Caballero, Brígido y Mellado, 2010; Blanco, Guerrero y Caballero, 2013; Caballero, Blanco y Guerrero, 2011; Furinghetti y Morselli, 2009; Zan, Bronw, Evans y Hannula, 2006 y Mellado, Blanco, Borrachero y Cárdenas, 2012).

Naranjo (2007) afirma que un estudiantado con alta autoestima tienden a participar más, a tener puntajes de competencia educativa más altos, a exhibir una conducta prosocial y a demostrar un logro académico mayor que el que tienen sus iguales con autoestima baja. Y que de esta manera:

(...) los éxitos continuos conducen a estas personas a una mayor estabilidad en la autoconfianza educativa; inversamente, aquellas personas que fracasan, experimentan una pérdida de autoestima, lo cual a su vez, contribuye a una continua carencia de éxito. La autoconfianza en el aprendizaje es la clave para ser exitoso en la educación (Naranjo, 2007, citado por Calderón, 2015, p. 7). 
Para Pérez-Tyteca (2012), con fundamento en Cretchley (2004), la tendencia de las investigaciones actuales sobre autoconfianza matemática es recalcar su importancia como un constructo de valor en la evaluación del aprendizaje de las matemáticas; sin embargo, no se conocen estudios en el ámbito costarricense sobre la autoconfianza matemática ni sobre la autoestima, por lo que esta investigación resulta pionera.

\section{MÉTODOS}

\section{Participantes}

Los participantes en el estudio fueron 2984 estudiantes ( $51 \%$ femenino) de sétimo a undécimo año de colegios públicos diurnos oficiales del Ministerio de Educación Pública de Costa Rica, matriculados en el 2015, previo permiso del Director de la institución. Los colegios participantes fueron seleccionados por muestreo simple aleatorio estratificado según la zona de ubicación (69.5\% urbano), y según la población por provincia (19.5\% Alajuela; $12.5 \%$ Cartago; 9.3\% Guanacaste; 10.6\% Heredia; 10.3\% Limón; 9.4\% Puntarenas; 28.4\% San José). Los estudiantes seleccionados para el estudio en cada colegio muestreado fueron los pertenecientes a la segunda sección de cada nivel (18.9\% sétimo; $20.5 \%$ octavo; $21.4 \%$ noveno; $19 \%$ décimo; $20.2 \%$ undécimo).

\section{Instrumentos}

La autoconfianza matemática fue medida con el test denominado Escala de Autoconfianza de FennemaSherman (1976) el cual contiene 12 ítems tipo Likert con cinco opciones desde "totalmente de acuerdo" a "totalmente en desacuerdo", el cual ha sido validado durante más de 30 años en diversas investigaciones (Nortes y Nortes, 2014; Berenguel, Gil, Montoro y Moreno, 2015).

La autoestima fue medida con base en la escala de autoestima de Rosenberg, la cual es una prueba estandarizada con 10 ítems tipo Likert con cinco opciones desde "totalmente de acuerdo" a "totalmente en desacuerdo" y que ha demostrado su validez y confiabilidad en múltiples estudios internacionales anteriores y con poblaciones de la mayor diversidad (Rojas-Barahona, Zegers y Forster, 2009; Vázquez, Jiménez y Vázquez-Morejón, 2004; Martín-Albo,
Nuñez, Navarro y Grijalbo, 2007; Vázquez, Vázquez-Morejón y Bellido, 2013).

Ambos tests son autoadministrados y cada estudiante responde de manera anónima y confidencial de acuerdo con sus creencias sobre sí mismos en cuanto a autoestima o confianza en matemática según lo afirmado en cada ítem.

\section{Procedimiento}

Se suministraron los tests descritos anteriormente a un grupo de cada nivel en cada colegio seleccionado, con una previa introducción seguida de las instrucciones correspondientes. En cada grupo, las respuestas fueron recolectadas en un formato de lápiz y papel en un tiempo aproximado de 15 minutos.

\section{Análisis estadísticos}

Con respecto a cada instrumento de medición, se realizó un estudio de sus características psicométricas: índice de discriminación, confiabilidad y unidimensionalidad; además se clasificaron los puntajes de los autoreportes de autoestima y autoconfianza matemática en cinco categorías: muy baja, baja, moderada, alta y muy alta.

Posteriormente, se contrastaron las siguientes tres hipótesis:

- Hipótesis 1: No existen diferencias en el nivel de autoconfianza matemática entre hombres y mujeres.

- Hipótesis 2: No existen diferencias en el nivel de autoestima entre hombres y mujeres.

- Hipótesis 3: No existe relación entre la autoconfianza matemática y la autoestima.

\section{RESULTADOS}

\section{Instrumento de medición}

Para el estudio de las características psicométricas de los instrumentos utilizados en este estudio, se determinó en primera instancia que los índices de discriminación obtenidos para cada ítem en la escala de autoconfianza $(\geq 0.504)$ son adecuados, por lo que no es necesario eliminar ningún ítem. Por otra parte, en la escala de autoestima, luego de eliminar el ítem 8 por tener un índice de discriminación no adecuado, los nueve restantes $(\geq 0.340)$ se mantienen para los análisis subsiguientes. 
De esta manera, el valor obtenido del estadístico denominado Alfa de Cronbach para la escala de autoconfianza fue $\alpha=0.899$ y para la escala de autoestima fue de $\alpha=0,781$, lo cual indica que ambos instrumentos son altamente confiables.

Posteriormente, se determinó que el instrumento de autoconfianza es unidimensional según el índice KMO cuyo valor fue de 0.926 con un valor $p<0.05$ y un primer factor que representa el $47.77 \%$ de la varianza total explicada. De igual manera, el instrumento de autoestima es unidimensional según el índice $\mathrm{KMO}$ cuyo valor fue de 0.813 con un valor $\mathrm{p}<0.05$ y un primer factor que representa el $38.31 \%$ de la varianza total explicada.

Otro hecho que comprueba la unidimensionalidad es que el valor del cociente entre la diferencia del primer y segundo autovalor, con la diferencia del segundo y tercer autovalor sea superior a 5. Para el instrumento de autoconfianza es 10.20 y para el de autoestima es 5.28, por lo que también se encontraron evidencias de la unidimensionalidad para ambos instrumentos.

Con respecto a los puntajes obtenidos para la autoconfianza matemática, en la tabla 1 se muestran los porcentajes según categoría:

Tabla 1. Nivel de autoconfianza matemática

\begin{tabular}{|c|c|}
\hline Muy baja & $0.9 \%$ \\
\hline Baja & $10.3 \%$ \\
\hline Moderada & $39.8 \%$ \\
\hline Alta & $39.1 \%$ \\
\hline Muy alta & $9.9 \%$ \\
\hline
\end{tabular}

Fuente: elaboración propia

Con respecto a los puntajes obtenidos para la autoestima matemática, en la tabla 2 se muestran los porcentajes según categoría:

Tabla 2. Nivel de autoestima matemática

\begin{tabular}{|c|c|}
\hline Muy baja & $0.2 \%$ \\
\hline Baja & $1.6 \%$ \\
\hline Moderada & $18.1 \%$ \\
\hline Alta & $56.3 \%$ \\
\hline Muy alta & $23.8 \%$ \\
\hline
\end{tabular}

\section{Contraste hipótesis 1}

Para el contraste de la primer hipótesis, se comparó la media de los hombres $(M=43.48, S D=8.85)$ con la media de las mujeres $(M=40.50, S D=9.38)$, y se encontró una diferencia estadísticamente significativa con un tamaño del efecto mediano ( $t$ $(2980.99)=8.93, p<0.05, d=0.33)$. Es decir, se rechaza la hipótesis nula y se acepta que existen diferencias en el nivel de autoconfianza matemática entre hombres y mujeres, pues en los hombres hay un nivel mayor de autoconfianza matemática.

\section{Contraste hipótesis 2}

Para el contraste de la segunda hipótesis, se comparó la media de los hombres $(M=36.42, S D=5.31)$ con la media de las mujeres $(M=35.63, S D=5.84)$, y se encontró una diferencia estadísticamente significativa con un tamaño del efecto bajo $(t(2973.19)=3.85$, $p<0.05, d=0,14)$. Es decir, se rechaza la hipótesis nula y se acepta que existen diferencias en el nivel de autoestima entre hombres y mujeres, pues en los hombres hay un nivel mayor de autoestima.

\section{Contraste hipótesis 3}

Para el contraste de la tercera hipótesis, se utilizó el coeficiente de correlación de Pearson entre las variables autoconfianza matemática y autoestima y se encontró una correlación estadísticamente significativa entre ambas variables $(r(2984)=0.37, p=0)$. Es decir, se rechaza la hipótesis nula y se acepta que existe relación entre la autoestima y la autoconfianza matemática.

\section{DISCUSIÓN}

La investigación se enfocó en establecer una relación entre la autoestima y la autoconfianza matemática en la educación secundaria oficial diurna costarricense, con el objetivo general de medir el nivel de ambos constructos y establecer si existen diferencias en la magnitud de cada uno por sexo.

Los resultados sugieren que, en forma conjunta, $78.9 \%$ de las y los estudiantes muestran niveles de autoconfianza entre alto y moderado y $74.4 \%$ de las y los estudiantes muestran niveles de autoestima entre alto y moderado.

Considerando que Pérez-Tyteca y Castro (2011) encontraron que los alumnos con más ansiedad 
matemática también presentan menor confianza en sus habilidades matemáticas, los hallazgos de la investigación son concordantes con los de Meza, Agüero y Suárez (2014), quienes en un estudio sobre ansiedad matemática en estudiantes de la educación media costarricense, es decir, en una población de características similares, encontraron que cerca del $70 \%$ de las y los estudiantes estaban en los niveles de ansiedad baja o media.

Los resultados de Meza, Agüero y Suárez (2014) sobre la ansiedad matemática resultaban sorprendentes en un país como Costa Rica en el que los niveles de aprobación en matemática no suelen ser buenos y en los que la aceptación de la matemática es baja. El hallazgo de esta nueva investigación confirma la situación, pues los niveles de autoconfianza matemática y autoestima encontrados se muestran más altos de los esperados.

Los hallazgos de estas dos investigaciones pueden ser tomados de manera positiva por las autoridades educativas costarricenses y por quienes se desempeñan directamente en las aulas, pero con cierta precaución, por cuanto en ambas los porcentajes de nivel medio (es decir, ni alto ni bajo) son relativamente importantes. En otros términos, si solo nos enfocamos en los porcentajes de autoconfianza alta, o en su caso en el de ansiedad matemática baja, los porcentajes rondan el 30\%, lo que reduce el panorama positivo de manera relevante.

La investigación también devela que las mujeres tienen niveles de autoconfianza matemática más bajos que los hombres, resultado que coincide con los obtenidos en otra investigación (Pérez-Tyteca, 2012), aunque tal como advierte esta autora, no todos los estudios coinciden en estos resultados y a menudo muestran inconsistencias.

Aunque no se han establecido relaciones causales para explicar estas diferencias, Fennema (1996, citada por Pérez-Tyteca, 2012), plantea que las discrepancias se deben a factores como el status económico, la etnicidad, la escuela o el profesor; es decir, tales diferencias entre hombres y mujeres en el nivel de autoconfianza matemática podrían estar explicadas por el contexto sociocultural y económico en que se desenvuelven, abriendo una interesante agenda de investigación en Costa Rica en donde no se han realizado estudios explicativos de las diferencias encontradas.

Considerando que la baja autoestima puede afectar el rendimiento académico en el o la estudiante universitaria, Cruz y Quiñones (2012) recomiendan a las y los docentes universitarios identificar y poner en práctica experiencias exitosas de otras universidades o de otros cursos para promover el rendimiento del estudiantado.

En este sentido, Calderón (2015) señala que "se debe considerar, por parte del o la docente, el uso del elogio y la restricción de palabras o gestos de censura. Asimismo, se debe practicar el respeto a las ideas del estudiante y utilizarlas en el desarrollo del tema o en la explicación de las dudas, realizar frecuentes interrogatorios generales e individuales sobre la materia objeto de aprendizaje, para cerciorarse de que el estudiante puede exponer en sus propias palabras lo que se le ha explicado. Además, el o la docente, debe comprobar y revisar los trabajos y ejercicios realizados, presentar los contenidos y realizar preguntas de forma clara. Debe demostrar entusiasmo por la labor educativa y formativa que se realiza, dedicando el tiempo suficiente para preparar las lecciones e impartirlas, revisar las pruebas y atender las dudas de las y los estudiantes, de manera que el estudiante perciba que no está solo en el proceso de enseñanza aprendizaje ya que su profesor está activamente comprometido por su aprendizaje" (pp.23-24).

Tales recomendaciones tienen como propósito fortalecer la autoestima de los estudiantes y esto obedece a que muchas de las propuestas de intervención educativa proponen una mejoría del rendimiento académico a partir del estímulo de la autoestima, "a través de un proceso psicológico complejo que involucra la percepción, imagen, estima y autoconcepto que se tiene" (Cruz y Quiñones, 2012, p. 27).

Los resultados sugieren, al menos, las siguientes líneas de investigación: profundizar en las causales de las diferencias detectadas en el nivel de autoconfianza matemática y de autoestima por sexo y de las que puedan explicar qué afecta el nivel de autoconfianza matemática y autoestima en los estudiantes de educación media, incorporando de manera explícita, como ha sugerido Gómez-Chacón (2010), el 
estudio de la realidad social que produce estas reacciones y el contexto sociocultural de los alumnos.

La investigación permite llegar a las siguientes conclusiones:

1. Cerca de un $78.9 \%$ del estudiantado muestra niveles de autoconfianza entre alto y moderado.

2. Existen diferencias en el nivel de autoconfianza matemática según el sexo, con niveles menores para las mujeres; sin embargo, la magnitud de esas diferencias es mediana.

3. Cerca de un $74.4 \%$ de la población muestra niveles de autoestima entre alto y moderado.

4. Existen diferencias en el nivel de autoestima según el sexo, con niveles menores para las mujeres; sin embargo, la magnitud de esas diferencias es baja.

5. Existe relación estadísticamente significativa entre la autoestima y la autoconfianza matemática.

Los resultados de la investigación permiten plantear, muy respetuosamente, las siguientes recomendaciones:

1. El desarrollo de investigaciones en las líneas sugeridas.

2. Complementar la investigación con un estudio de tipo explicativo que permita avanzar en la identificación de causales de la autoconfianza matemática y de la autoestima en la educación media.

3. Ampliar el rango de la investigación replicando el estudio en colegios privados o nocturnos, pues este tipo de instituciones no fueron consideradas en la investigación.

\section{AGRADECIMIENTOS}

Se agradece el apoyo de la Vicerrectoría de Investigación y Extensión del Instituto Tecnológico de Costa Rica y a los colegios participantes.

\section{REFERENCIAS BIBLIOGRÁFICAS}

Berenguel, E., Gil, F., Montoro, A.B. y Moreno, M. (2015). Influencia de la autoconfianza y el perfil motivacional en el "flujo" en matemáticas. En C. Fernández, M. Molina y N. Planas (Eds.), Investigación en Educación Matemática XIX (pp. 173-
181). Alicante: SEIEM. Recuperado de http://rua. ua.es/dspace/bitstream/10045/51386/1/2015Actas-XIX-SEIEM_15.pdf, el 12 de marzo de 2015.

Calderón, M. (2015). Relación entre la autoestima y el rendimiento académico en los cursos de formación matemática de las carreras de ingeniería en el ITCR. (Tesis inédita de doctorado). Universidad de Valencia, España.

Córdova, C. (2010). La autoestima y su influencia en el rendimiento académico de los niños/as de segundo, tercero y cuarto año de educación básica de la escuela "Amazonas" del cantón CevaIlos, caserío La Florida, en el período diciembre 2009 a febrero del 2010. Tesis de licenciatura. Universidad Técnica de Ambato, Ecuador. Recuperado de http://repositorio.uta.edu.ec/handle/123456789/637 el el 15 de marzo de 2015.

Cruz, F. y Quiñones, A. (2012). Autoestima y rendimiento académico en estudiantes de enfermería de Poza Rica, Veracruz, México. Revista Unipluri/versidad, 12(1), 25-35. Recuperado de: http://aprendeenlinea.udea.edu.co/revistas/ index.php/unip/article/view/13275/11894 el 10 de febrero de 2015.

Erazo, N. (2013). La autoestima y su repercusión en el rendimiento académico de los estudiantes de los terceros grados de educación básica de la escuela José Reyes de la ciudad de San Gabriel, provincia del Carchi. Tesis de licenciatura Facultad de Ciencias Humanas y de la Educación Universidad de Ambato, Ecuador. Recuperado de http://repositorio.uta.edu.ec/bitstream/123456789/6430/1/FCHE_LEB_1148.pdf el 25 de abril de 2015.

Fernández, O., Martínez-Conde, M. y MelipiIlán, R. (2009). Estrategias de Aprendizaje y Autoestima. Su relación con la permanencia y deserción universitaria. Estudios Pedagógicos XXXV (1), 27-45. Recuperado de http://www.scielo.cl/scielo.php?pid=S071807052009000100002\&script=sci_arttext el 24 de abril de 2015.

Gamboa, R. (2012). ¿Equidad de género en la enseñanza de las Matemáticas? Revista Electrónica Educare, 16(1), 63-78. 
Relación entre autoestima y autoconfianza matemática en estudiantes de educación media costarricense

Recuperado de http://www.redalyc.org/ pdf/1941/194124281007.pdf el 18 de mayo de 2015.

García, E. (2012). Relación de los factores, autoestima, motivación, puntaje de ingreso en el rendimiento académico de los alumnos ingresantes 2010, a la Universidad Nacional de Tumbes. (Tesis de Maestría). Universidad Nacional Mayor de San Marcos, Perú. Recuperado de

http://cybertesis.unmsm.edu.pe/bitstream/cybertesis/2903/1/Garc\%C3\%ADa_ge.pdf el 4 de abril de 2015.

Gil, N., Blanco, L. y Guerrero, B. (2006). El papel de la afectividad en la resolución de problemas matemáticos. Revista de educación, (340), 551569. Recuperado de http://www.revistaeducacion.mec.es/re340/re340_20.pdf el 25 de mayo de 2015.

Gómez-Chacón, I. (2010). Actitudes de los estudiantes en el aprendizaje de la matemática con tecnología. Enseñanza de las ciencias, 28(2), 227-244. Recuperado de http://eprints.ucm. es/21500/1/IGomez1.pdf el 5 de mayo de 2015.

Martín-Albo, J., Nuñez, J., Navarro, J. y Grijalbo, F. (2007) The Rosenberg Self-Esteem Scale: Traslation and Validation in University Students. The Spanish Journal of Psychology, 10 (2), 408-467. Recuperado de http://revistas.ucm.es/indexphp/ SJOP/article/view.SJOP0707220458A/28907 el 5 de mayo de 2015.

Mellado, V., Blanco, A., Borrachero, A. y Cárdenas, J. (2012) Las emociones en la enseñanza y el aprendizaje de las ciencias y las matemáticas. Madrid, España: Editorial DEPROFE. Recuperado de

www.eweb.unex.es/web/dcem/VOLUMEN\%20 Ilok.pdf el 6 de junio de 2015.

Meza, L. G., Agüero, E. y Suárez, Z. (2014). ESAM: Estudio de la ansiedad matemática en la educación media. (Informe final de proyecto de investigación). Cartago, CR: Instituto Tecnológico de Costa Rica. Recuperado de http://repositoriotec. tec.ac.cr/handle/2238/4733 el 3 de marzo de 2015.
Mezerville, G. (2005). Ejes de salud mental: Los procesos de autoestima, dar y recibir afecto y adaptación al estrés. Madrid, España: Editorial Trillas

Naranjo, M. L. (2007). Autoestima: un factor relevante en la vida de la persona y tema esencial del proceso educativo. Revista Electrónica Actualidades Investigativas en Educación, (7), 1-27. Recuperado de

http://revele.com.veywww.redalyc.org/articulo. oa?id=44770311 el 23 de enero de 2015.

Nortes, R. y Nortes, A. (2014). ¿Tienen ansiedad hacia las matemáticas los futuros matemáticos? Profesorado. Revista de currículo y formación del profesorado, 18(2), 153-170. Recuperado de http:// www.redalyc.org/articulo.oa?id=56732350009 el 2 de febrero de 2015.

Pérez-Tyteca, P. y Castro, E. (2011). La ansiedad matemática y su red de influencias en la elección de carrera Universitaria. En M. Marín, G. Fernández, L. Blanco y M.M. Palarea (Eds.), Investigación en Educación Matemática XV (pp. 471-480). Ciudad Real: SEIEM. Recuperado de http://funes.uniandes.edu.co/1831/ el 12 de abril de 2015.

Pérez-Tyteca, P. (2012). La ansiedad matemática como centro de un modelo causal predictivo de la elección de carreras. (Tesis inédita de doctorado). Universidad de Granada, España.

Pérez-Tyteca, P., Monje, J., y Castro, E. (2013). Afecto y matemáticas. Diseño de una entrevista para acceder a los sentimientos de alumnos adolescentes. Avances de Investigación en Educación Matemática, 4, 65-82. Recuperado de http://www.aiem.es/index.php/aiem/article/ view/55/29 el 12 de abril de 2015.

Primi, C., Busdraghi, C., Tomasetto, C., Morsanyi, K. y Chiesi, F. (2014). Measuring math anxiety in Italian college and high school students: validity, reliability and gender invariance of the Abbreviated Math Anxiety Scale (AMAS). Learning and Individual Differences, 34, 51-56. doi:10.1016/j. lindif.2014.05.012

Rabell, M. (2012). Autoestima y rendimiento académico: un estudio aplicado al aula de educación primaria. Tesis de Maestría. Facultad 
de Educación Universidad Internacional de La Rioja, Barcelona, España. Recuperado de http:// reunir.unir.net/handle/123456789/1009 el 5 de julio de 2015.

Rojas-Barahona, C., Zegers, B. y Förster, C. (2009). La escala de autoestima de Rosenberg: Validación para Chile en una muestra de jóvenes adultos, adultos y adultos mayores. Revista Médica Chile, 137, 791-800. Recuperado de http://www.scielo.cl/scielo.php?pid=S0034$98872009000600009 \&$ script=sci_arttext eñ 13 de febrero de 2015.

Schnell, K., Ringeisen, T., Raufelder, D. y Rohrmann, S. (2015). The impact of adolescents' self-efficacy and self-regulated goal attainment processes on school performance - Do gender and test anxie- ty matter? Learning and Individual Differences, 38, 90-98. doi:10.1016/j.lindif.2014.12.008.

Vázquez, A., Jiménez, R. y Vázquez-Morejón, R. (2004). Escala de autoestima de Rosenberg: fiabilidad y validez en población clínica española. Apuntes de Psicología, 22(2), 247-255.

Vázquez A., Vázquez-Morejón, R. y Bellido, G. (2013). Fiabilidad y validez de la Escala de Autoestima de Rosenberg (EAR) en pacientes con diagnóstico de psicosis. Apuntes de Psicología, 21(1), 37-43. Recuperado de http://apuntesdepsicologia.es/index.php/revista/article/ view/296/277 el 15 de julio de 2015. 